\title{
Clinicopathologic characteristics and prognosis of gastroenteropancreatic neuroendocrine neoplasms: a multicenter study in South China
}

Cheng Fang ${ }^{1 \dagger}$, Wei Wang ${ }^{1 \dagger}$, Yu Zhang ${ }^{2 \dagger}$, Xingyu Feng ${ }^{3 \dagger}$, Jian Sun ${ }^{4 \dagger}$, Yujie Zeng ${ }^{5 \dagger}$, Ye Chen ${ }^{6 *}$, Yong $\mathrm{Li}^{3^{*+}}$, Minhu Chen ${ }^{2}$, Zhiwei Zhou ${ }^{1 *+}$ and Jie Chen ${ }^{2^{*}+}$

\begin{abstract}
Background: Gastroenteropancreatic neuroendocrine neoplasms (GEP-NENs) are a heterogeneous group of rare tumors. Many issues in terms of epidemiologic features, pathogenesis, and treatment of GEP-NENs are still under discussion. Our study aimed to analyze the clinicopathologic characteristics and prognosis of Chinese patients with GEP-NENs.

Methods: Complete clinicopathologic data and survival information of 1183 patients with GEP-NENs treated between 2005 and 2015 were collected from five medical centers in Guangdong Province, China. Patient survival was estimated using the Kaplan-Meier method and analyzed using the log-rank test; prognostic factors were analyzed using the Cox proportional hazards model.

Results: The most common tumor location was the rectum (37.4\%), followed by the pancreas (28.1\%), stomach (20.7\%), small intestine (7.2\%), appendix (3.4\%), and colon (3.3\%). After initial definitive diagnosis, 1016 (85.9\%) patients underwent surgery. The 1-, 3-, and 5-year overall survival (OS) rates for the entire cohort were $87.9 \%, 78.5 \%$, and $72.8 \%$, respectively. The 3-year OS rates of patients with G1, G2, and G3 tumors were $93.1 \%, 82.7 \%$, and $43.1 \%$, respectively $(P<0.001)$. The 3-year OS rates of patients with stage I, II, III, and IV tumors were $96.0 \%, 87.3 \%, 64.0 \%$, and $46.8 \%$, respectively $(P<0.001)$. Patients with distant metastasis who underwent palliative surgery had a longer survival than those who did not $(P=0.003)$. Similar survival benefits of palliative surgery were observed in patients
\end{abstract}

\footnotetext{
*Correspondence: yechen@smu.edu.cn; yuan821007@126.com;

zhouzhw@sysucc.org.cn; chen0jie@hotmail.com

${ }^{\dagger}$ Cheng Fang, Wei Wang, Yu Zhang, Xingyu Feng, Jian Sun, Yujie Zeng, Ye

Chen, Yong Li, Zhiwei Zhou and Jie Chen contributed equally to this work

1 Department of Gastric Surgery, Sun Yat-sen University Cancer Center,

State Key Laboratory of Oncology in South China, Collaborative

Innovation Center for Cancer Medicine, 651 Dongfeng Road East,

Guangzhou 510060, Guangdong, P. R. China

${ }^{2}$ Department of Gastroenterology, The First Affiliated Hospital of Sun Yat-

sen University, Guangzhou 510080, Guangdong, P. R. China

${ }^{3}$ Department of General Surgery, Guangdong General Hospital,

Guangdong Academy of Medical Science, Guangzhou 510080,

Guangdong, P. R. China

${ }^{6}$ Department of Gastroenterology, Nanfang Hospital of Southern Medical

University, Guangdong Provincial Key Laboratory of Gastroenterology,

Guangzhou 510515, Guangdong, P. R. China

Full list of author information is available at the end of the article
} 
with neuroendocrine tumor $(P=0.031)$ or neuroendocrine carcinoma $(P=0.046)$. In multivariate analysis, age, grade, $\mathrm{N}$ category, M category, and surgery were found to be independent prognostic factors.

Conclusions: Patients with GEP-NENs who are women, younger than 50 years old, have smaller tumor size, have lower tumor grade, have lower T/N/M category, and who undergo surgery can have potentially longer survival time. Our data showed that surgery can improve the prognosis of GEP-NEN patients with distant metastasis. However, randomized controlled trials need to be conducted to establish the optimal criteria for selecting patients to undergo surgery.

Keywords: Gastroenteropancreatic neuroendocrine neoplasms, Surgery, Prognosis, China

\section{Background}

Neuroendocrine neoplasms (NENs) are a heterogeneous group of rare tumors with different and complex clinical behaviors, originating from peptidergic neurons and neuroendocrine cells throughout the body, and most are gastroenteropancreatic neuroendocrine neoplasms (GEP-NENs) [1]. Over the years, the nomenclature and classification of NENs have undergone significant changes. In 1907, Oberndorfer [2] first described it as a benign "carcinoid" tumor. In 2000, the term "neuroendocrine tumors" was officially used in the World Health Organization (WHO) classification to more accurately depict their malignant potential. In 2010, the Ki-67 proliferative index and mitotic count were first used in the WHO classification as important diagnostic and prognostic factors for NENs [3]. The current staging systems developed by the American Joint Committee on Cancer/ Union for International Cancer Control (AJCC/UICC) and European Neuroendocrine Tumor Society (ENETS) are both based on the TNM staging system.

To data, only a few studies, based on data from national cancer registries-mainly from the United States [4], Norway [5], England [6], Spain [7], Germany [8], and Korea [9] —have showed epidemiologic features of NENs. However, for some of these studies, the current staging system and diagnostic criteria were not used, or detailed demographic and clinicopathologic characteristics or therapeutic intervention information were lacking. Generally, the incidence of GEP-NENs has increased continuously worldwide over the last decades. In patients from Western countries, primary tumors locate mostly in the small intestine, rectum, and pancreas [4-7]. Furthermore, the distribution of tumor stages and patients' overall survival (OS) rates are discrepant in different countries. Although several single-center studies with limited sample size on the clinicopathologic features of GEP-NENs have been performed in Chinese populations $[10,11]$, a multicenter study with a large population has been needed to fully understand this rare tumor and identify optimal therapeutic strategies. In 2010, several large centers in Guangdong Province, China, began a long-term collaboration focused on providing multidisciplinary treatments of NENs. The present study aimed to analyze clinicopathologic characteristics and prognosis of GEP-NEN patients from these centers in South China.

\section{Patients and methods \\ Patient selection}

We collected data from the case management systems of five medical centers in Guangdong Province, China: Sun Yat-sen University Cancer Center, the First Affiliated Hospital of Sun Yat-sen University, Guangdong General Hospital, Nanfang Hospital of Southern Medical University, and Sun Yat-sen Memorial Hospital of Sun Yatsen University. We included only patients who (1) were histopathologically diagnosed with GEP-NENs; (2) were treated and followed between January 2005 and December 2015; and (3) had complete medical records containing demographic data, clinicopathologic data, and follow-up results. Patients with additional synchronous or metachronous malignancies were excluded.

The present study was approved by the ethics committees of the five hospitals and complied with the Declaration of Helsinki.

\section{Diagnosis, staging, and treatment}

GEP-NENs were histopathologically defined according to the current WHO 2010 classifications [3]; for patients diagnosed and treated before 2010, tumors were redefined. Functional or nonfunctional GEP-NENs were defined according to whether the patients presented with clinical symptoms caused by hormones. The tumor locations of GEP-NENs were categorized as the stomach, pancreas, small intestine (including the duodenum, jejunum, and ileum), appendix, colon, and rectum. Pathologic examinations of the sections from endoscopic biopsies, intraoperative incisional biopsies, and resected gross specimens were performed by pathologists following diagnostic criteria which contained typical morphological findings and immunohistochemical staining of neuroendocrine markers, including chromogranin A and synaptophysin. Based on the WHO 2010 classifications [3], G1, G2, and G3 grading levels were classified 
according to the Ki-67 index ( $\leq 2 \%, 3 \%-20 \%$, and $>20 \%)$ and mitotic rates $(<2$ per $10 \mathrm{HPF}$ [high power field], $2-20$ per $10 \mathrm{HPF}$, and $>20$ per $10 \mathrm{HPF}$ ). If the grade of Ki-67 index was not in agreement with the grade of mitotic rate, the parameter with the higher grade was used for classification. According to different degrees of pathologic differentiation, all NENs were divided into well-differentiated neuroendocrine tumors (NETs), poorly differentiated neuroendocrine carcinomas (NECs), and mixed adenoneuroendocrine carcinomas (MANECs) which contained two components (adenocarcinoma and neuroendocrine neoplasms). Based on clinical, pathologic, and imaging data, the tumors were classified into stages I, II, III, and IV according to the ENETS TNM classification $[12,13]$. Therapeutic modalities included surgeries such as endoscopic resection (endoscopic mucosal resection or endoscopic submucosal dissection), radical resection, and debulking or cytoreductive surgery and systematic therapies such as chemotherapy and somatostatin analogues or targeted drugs.

\section{Follow-up}

Follow-up data were based on outpatient records and on messaging and/or telephonic interviews conducted between the day patients were discharged and March 31, 2016. A strict program of disease monitoring including electronic gastroduodenoscopic examinations, abdominal ultrasonography (and computed tomography, if necessary), chest X-rays, and blood examinations, was conducted, if clinically required. Recommended followup intervals were 6 months. OS was defined as the duration from the date of pathologic diagnosis to the date of death or the last follow-up.

\section{Statistical analysis}

Data were analyzed using the SPSS software version 18.0 (SPSS Inc., Chicago, IL, USA). Categorical variables were analyzed using the Chi-square test. Kaplan-Meier analysis was used to estimate the cumulative OS rate; log-rank test was used to analyze significances among the different groups; and the Cox proportional hazards model was used for multivariate analysis. The two-tailed $P$ values less than 0.05 were considered statistically significant.

\section{Results}

\section{Demographic data}

A total of 1496 patients were included. Of these, 1183 patients were selected into our study, since 105 patients were lost during follow-up, 182 did not have complete clinicopathologic data, and 26 had other types of malignancies and were thus excluded. The selected patients included 482 (40.7\%) from Sun Yat-sen University Cancer Center, 309 (26.1\%) from the First Affiliated Hospital of Sun Yat-sen University, 143 (12.1\%) from Guangdong General Hospital, 131 (11.1\%) from Nanfang Hospital of Southern Medical University, and 118 (10.0\%) from Sun Yat-sen Memorial Hospital of Sun Yat-sen University. There were 715 men and 468 women in a ratio of 1.5:1, with a median age of 51 years (range, 9-87 years).

\section{Clinicopathologic factors}

Clinicopathologic characteristics of these 1183 GEPNEN patients are shown in Table 1. Nonfunctional tumors accounted for $82.8 \%$ (979) of GEP-NENs; the remaining $17.2 \%$ (204) were functional tumors. The most common tumor location was the rectum, followed by the pancreas, stomach, small intestine, appendix, and colon. The median diameter of the primary tumor was $2.0 \mathrm{~cm}$ (range, 0.1-20.0 cm), based on postoperative resection specimens or imaging examinations before surgery. Among all cases, 608 (51.4\%) were G1, 270 (22.8\%) were G2, and 305 (25.8\%) were G3. Well-differentiated NETs accounted for $74.5 \%$ (881). Based on ENETS criteria, 479 cases $(40.5 \%)$ were stage I, 246 (20.8\%) were stage II, 200 (16.9\%) were stage III, and $258(21.8 \%)$ were stage IV. In this study, $85.9 \%$ (1016) of the patients underwent surgery (radical or palliative resection). In addition, systematic treatment was administered to 306 (25.9\%) patients. Chi-square analyses for the variables in the surgery and non-surgery groups of patients with M1 category tumors showed that the clinicopathologic characteristics between the two groups were not significantly different, except for tumor size and location (Table 2). In the surgery group, more patients had tumors located in the stomach and intestine and with tumor sizes smaller than or equal to $4.0 \mathrm{~cm}$ compared with the non-surgery group.

\section{Patient survival}

The median OS for GEP-NEN patients was 28 months (range, 4-135 months). The 1-, 3-, and 5-year OS rates were $87.9 \%, 78.5 \%$, and $72.8 \%$, respectively. The OS curves stratified by gender, age, tumor functionality, tumor location, tumor size, grade, tumor type, $\mathrm{T}$ category, $\mathrm{N}$ category, $\mathrm{M}$ category, TNM stage, and treatment are displayed in Fig. 1. The 3-year OS rates for patients with TNM stage I, II, III, and IV diseases were $96.0 \%, 87.3 \%, 64.0 \%$, and $46.8 \%$, respectively $(P<0.001)$. The 3-year OS rates for patients with G1, G2, and G3 diseases were $93.1 \%, 82.7 \%$, and $43.1 \%$, respectively $(P<0.001)$. With respect to tumor locations, the 3-year OS rates for patients with tumors in the rectum, appendix, small intestine, pancreas, stomach, and colon were $90.2 \%, 86.0 \%, 75.8 \%, 75.3 \%, 64.6 \%$, and $48.5 \%$, respectively (Table 3 ). The 3-year OS rate of patients with M0 category tumors who underwent surgery was higher than that of those who did not $(88.7 \%$ vs. $45.2 \%, P<0.001)$ 
Table 1 Clinicopathologic characteristics of 1183 patients with gastroenteropancreatic neuroendocrine neoplasms (GEP-NENs)

\begin{tabular}{|c|c|}
\hline Variable & No. of cases (\%) \\
\hline \multicolumn{2}{|l|}{ Gender } \\
\hline Female & $468(39.6)$ \\
\hline Male & $715(60.4)$ \\
\hline \multicolumn{2}{|l|}{ Age (years) } \\
\hline$\leq 50$ & $564(47.7)$ \\
\hline$>50$ & $619(52.3)$ \\
\hline \multicolumn{2}{|c|}{ Tumor functionality } \\
\hline Nonfunctional & $979(82.8)$ \\
\hline Functional & $204(17.2)$ \\
\hline \multicolumn{2}{|l|}{ Tumor location } \\
\hline Stomach & $245(20.7)$ \\
\hline Pancreas & $332(28.1)$ \\
\hline Small intestine & $85(7.2)$ \\
\hline Appendix & $40(3.4)$ \\
\hline Colon & $39(3.3)$ \\
\hline Rectum & $442(37.4)$ \\
\hline \multicolumn{2}{|l|}{ Tumor size (cm) } \\
\hline$<2.0$ & $616(52.1)$ \\
\hline $2.0-4.0$ & $272(23.0)$ \\
\hline$>4.0$ & $295(24.9)$ \\
\hline \multicolumn{2}{|c|}{ Grade (WHO 2010 classification) } \\
\hline G1 & $608(51.4)$ \\
\hline $\mathrm{G} 2$ & $270(22.8)$ \\
\hline $\mathrm{G} 3$ & $305(25.8)$ \\
\hline \multicolumn{2}{|l|}{ Tumor type } \\
\hline NET & $881(74.5)$ \\
\hline NEC & $261(22.1)$ \\
\hline MANEC & $41(3.5)$ \\
\hline \multicolumn{2}{|c|}{ T category (ENETS criteria) } \\
\hline $\mathrm{T} 1$ & $510(43.1)$ \\
\hline $\mathrm{T} 2$ & $220(18.6)$ \\
\hline $\mathrm{T} 3$ & $314(26.5)$ \\
\hline $\mathrm{T} 4$ & $139(11.7)$ \\
\hline \multicolumn{2}{|c|}{ N category (ENETS criteria) } \\
\hline No & $846(71.5)$ \\
\hline N1 & $337(28.5)$ \\
\hline \multicolumn{2}{|c|}{ M category (ENETS criteria) } \\
\hline M0 & $925(78.2)$ \\
\hline M1 & $258(21.8)$ \\
\hline \multicolumn{2}{|c|}{ TNM stage (ENETS criteria) } \\
\hline 1 & $479(40.5)$ \\
\hline$\|$ & $246(20.8)$ \\
\hline III & $200(16.9)$ \\
\hline IV & $258(21.8)$ \\
\hline \multicolumn{2}{|l|}{ Surgery } \\
\hline Yes & $1016(85.9)$ \\
\hline No & $167(14.1)$ \\
\hline
\end{tabular}

WHO World Health Organization, NET neuroendocrine tumor, NEC neuroendocrine carcinoma, MANEC mixed adenoneuroendocrine carcinoma, ENETS European Neuroendocrine Tumor Society
(Fig. 2a). Interestingly, patients with M1 category tumors who underwent palliative surgery had a longer median OS duration compared with those who did not undergo palliative surgery (51 months vs. 17 months, $P=0.003$ ) (Fig. 2b). Furthermore, stratified analyses showed similar survival benefits for both patients with NETs (median OS: not reached vs. 39 months, $P=0.031$ ) (Fig. 2c) and patients with NECs (median OS: 17 months vs. 11 months, $P=0.046$ ) (Fig. $2 \mathrm{~d}$ ).

\section{Univariate and multivariate analyses}

To determine the prognostic factors of OS, multivariate analysis using the Cox proportional hazards model with a forward stepwise model was performed for the 1183 cases of GEP-NENs. Gender, age, tumor functionality, tumor location, tumor size, grade, tumor type, $\mathrm{T}$ category, N category, M category, TNM stage, and treatment were included in the univariate analysis (Table 3). The results showed that all the above factors, except for tumor functionality, were significantly associated with prognosis. Moreover, when these significant variables were entered into the Cox proportional hazards model, age, grade, $\mathrm{N}$ category, $\mathrm{M}$ category, and treatment were found to be independent prognostic factors (Table 3).

\section{Discussion}

In this study, we collected and analyzed the clinicopathologic features of 1183 GEP-NEN patients with complete follow-up data from five large hospitals in South China. Our data showed the most common locations of primary tumor were the rectum, pancreas, and stomach. By multivariate analysis, age, grade, $\mathrm{N}$ category, $\mathrm{M}$ category, and treatment were found to be independent prognostic factors. Of note, our findings have shown that patients with distant metastasis who underwent palliative surgery had a longer survival time than those who did not undergo palliative surgery.

Until recently, only four studies containing more than 1000 GEP-NEN cases were reported worldwide [5, 6, 14, 15]. As with other studies [15-17], we reported the gender ratio (male vs. female, 1.5:1) and median age (51 years) at diagnosis; and found most of the GEPNENs in our study were nonfunctional and were found by incidental diagnosis, physical examination, or nonspecific symptoms, such as abdominal pain, gastrointestinal bleeding, diarrhea, and jaundice, which are similar to previous studies $[18,19]$. The distribution of tumor locations of GEP-NENs that showed in our study was similar to those found in several other Asian studies $[9,11]$. In most reports from the United States and European countries-typically those with data derived from the National Cancer Institute's Surveillance, Epidemiology and End Results (SEER) database [4], the National Cancer 
Table 2 Chi-square analysis for clinicopathologic variables in patients with M1 category GEP-NENs who did or did not undergo surgery

\begin{tabular}{|c|c|c|c|}
\hline Variable & $\begin{array}{l}\text { Surgery group } \\
\text { [cases (\%)] }\end{array}$ & $\begin{array}{l}\text { Non-surgery group } \\
\text { [cases }(\%)]\end{array}$ & $P$ value \\
\hline Total & 127 & 131 & \\
\hline Gender & & & 0.233 \\
\hline Male & $93(73.2)$ & $87(66.4)$ & \\
\hline Female & $34(26.8)$ & 44 (33.6) & \\
\hline Age (years) & & & 0.403 \\
\hline$\leq 50$ & $52(40.9)$ & $47(35.9)$ & \\
\hline$>50$ & $75(59.1)$ & $84(64.1)$ & \\
\hline Tumor functionality & & & 0.144 \\
\hline Nonfunctional & $112(88.2)$ & $107(81.7)$ & \\
\hline Functional & $15(11.8)$ & $24(18.3)$ & \\
\hline Tumor location & & & $<0.001$ \\
\hline Stomach & $39(30.7)$ & $29(22.1)$ & \\
\hline Pancreas & $33(26.0)$ & $70(53.4)$ & \\
\hline Small intestine & $17(13.4)$ & $8(6.1)$ & \\
\hline Appendix & $1(0.8)$ & $0(0.0)$ & \\
\hline Colon & $10(7.9)$ & $4(3.1)$ & \\
\hline Rectum & $27(21.2)$ & $20(15.3)$ & \\
\hline Tumor size (cm) & & & 0.006 \\
\hline$<2.0$ & $25(19.7)$ & $14(10.7)$ & \\
\hline $2.0-4.0$ & $50(39.4)$ & $38(29.0)$ & \\
\hline$>4.0$ & $52(40.9)$ & $79(60.3)$ & \\
\hline Grade & & & 0.743 \\
\hline G1 & $25(19.7)$ & $24(18.3)$ & \\
\hline $\mathrm{G} 2$ & $46(36.2)$ & $43(32.8)$ & \\
\hline G3 & $56(44.1)$ & $64(48.9)$ & \\
\hline Tumor type & & & 0.574 \\
\hline NET & $70(55.1)$ & 65 (49.6) & \\
\hline NEC & $52(40.9)$ & $62(47.3)$ & \\
\hline MANEC & $5(3.9)$ & $4(3.1)$ & \\
\hline
\end{tabular}

NET neuroendocrine tumor, NEC neuroendocrine carcinoma, MANEC mixed adenoneuroendocrine carcinoma

Registry of Gastroenteropancreatic Neuroendocrine Tumors (RGENE) [6], and the Norwegian Registry of Cancer (NRC) [5] -the rectum, small intestine, and pancreas were the most common NEN locations, whereas gastric NENs accounted for less than 10\% of GEP-NEN cases. However, in the present study, the rectum, pancreas, and stomach were the most common locations of NENs, whereas small intestine NENs accounted for less than $10 \%$. We consider that racial disparities may contribute to the distinctions in distributions of tumor locations. Furthermore, the inconsistencies may also be due to the high incidence of gastric cancer in China [20,21], which has raised health awareness and encouraged more cautious people to have regular physical examinations to identify gastric diseases. All specimens in the current analysis were reviewed and graded according to the latest nomenclature and classification system for GEPNENs. In our study, G1 tumors accounted for $51.4 \%$, which was consistent with the results of studies from Korea [9], Austria [22], and the Netherlands [23]. Based on the ENETS staging system, lymph node and/or distant metastasis occurred in $38.7 \%$ of patients, which was lower than the occurrence rates reported in Spain (44.2\%) by Garcia-Carbonero et al. [7] and Hong Kong, China (53.4\%) by Rothenstein et al. [24]. The 5-year OS rate in our entire cohort was $72.8 \%$, which was higher than those in cohorts based on the SEER registries from the United States (50\%) [4] and NRC registries from Norway (59\%) [5], and similar to those in some cohorts from European countries $(75 \%-79 \%)$ [6-8]. The discrepancy with data from SEER and NRC may be attributed to the high ratio of NEC patients in the SEER database [4] as well as racial 


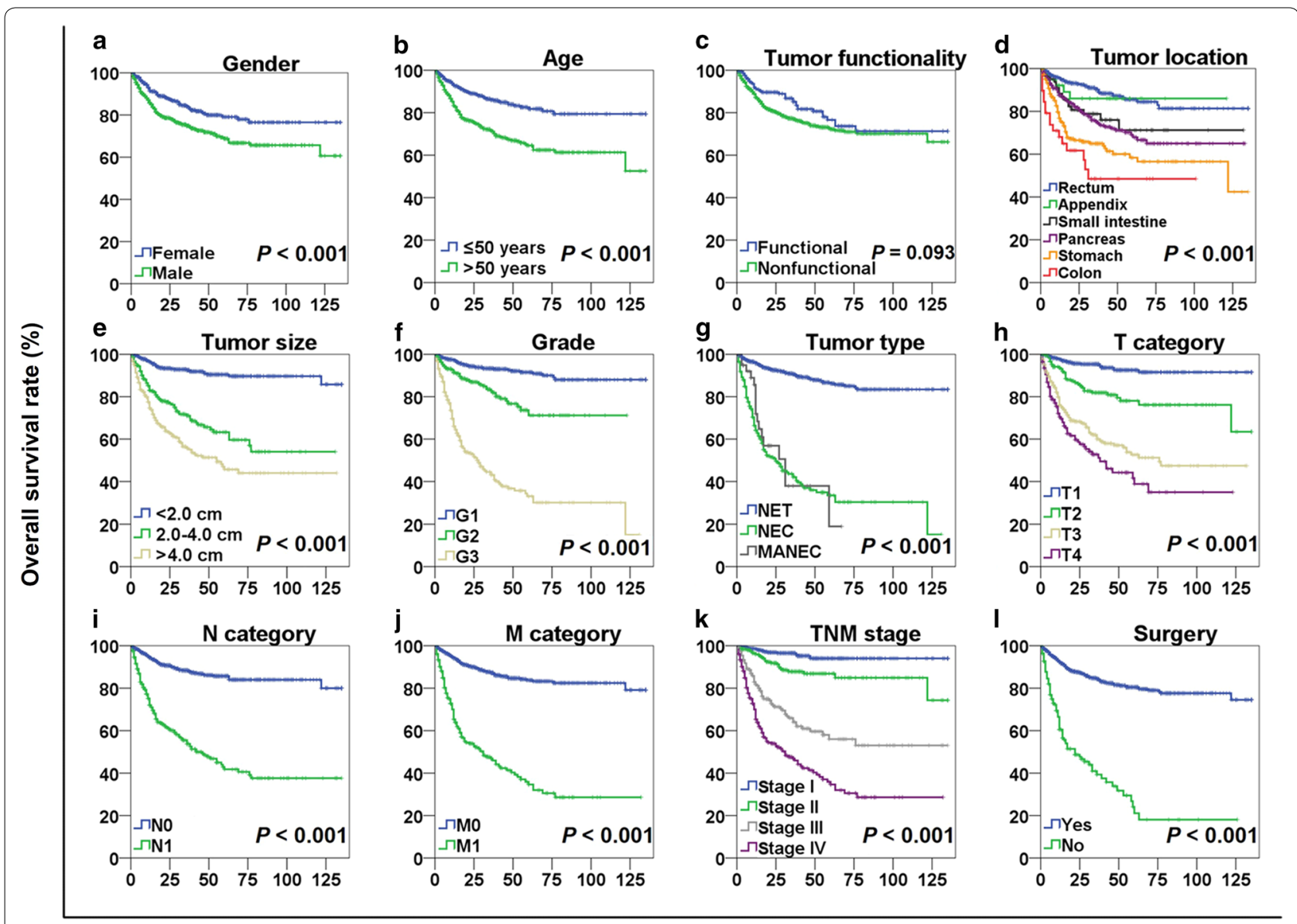

Time after diagnosis (months)

Fig. 1 Overall survival (OS) curves stratified by different variables in 1183 patients with gastroenteropancreatic neuroendocrine neoplasms

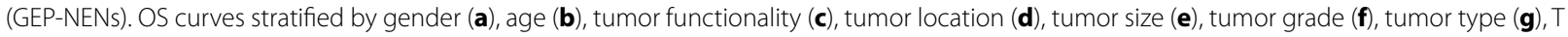
category $(\mathbf{h})$, N category $(\mathbf{i})$, M category $(\mathbf{j})$, TNM stage $(\mathbf{k})$, and surgery (I). OS was different among subgroups stratified by all variables (all $P<0.001$ ) except tumor functionality $(P=0.093)$. NET neuroendocrine tumor, NEC neuroendocrine carcinoma, MANEC mixed adenoneuroendocrine carcinoma

and geographic differences in the NRC database [5]. More studies across different continents and countries to compare these clinicopathologic characteristics and prognosis may be required to better understand GEP-NENs.

In our univariate analysis, women and younger patients with smaller tumors, lower tumor grade, less invasion, and surgical treatment and those without lymph node or distant metastasis had significantly longer OS (all $P<0.001$ ). Gender was not a prognostic factor in several studies [5-8], and only one study showed a survival benefit for female patients [17]. Interestingly, in our study, patients with distant metastasis who underwent palliative surgery had a longer OS than those who did not undergo palliative surgery (median OS: 51 months vs. 17 months, $P=0.003$ ). Our results showed that many patients with distant metastasis whose tumors were located in the stomach and intestine and who had tumor sizes less than or equal to $4 \mathrm{~cm}$ underwent surgery. This phenomenon may be partly due to higher rates of preoperative symptoms, such as massive gastrointestinal bleeding and obstruction, which required emergent surgery. Also, compared with pancreatic surgeries, gastrointestinal surgeries showed lower rates of postoperative complications [25-27]. Moreover, patients with smaller tumor sizes were possibly considered to have lower tumor burden and were more likely to undergo surgery. Different treatments were selected in consideration of discrepancies of tumor location and size, which may account for the survival difference among patients with distant metastasis. In addition, analysis stratified by tumor types showed that patients with distant metastasis of NETs (similar to G1/G2 tumors with distant metastasis) and NECs (similar to G3 tumors with distant metastasis) could benefit from surgery ( $P=0.031$ and $P=0.046$, respectively), which has not been reported in any other study. On the 
Table 3 Univariate and multivariate analyses of prognostic factors for overall survival of 1183 patients with GEP-NENs

\begin{tabular}{|c|c|c|c|c|c|c|c|c|}
\hline \multirow[t]{2}{*}{ Variable } & \multirow[t]{2}{*}{ 3-year OS rate (\%) } & \multicolumn{2}{|c|}{ Univariate analysis } & \multicolumn{5}{|c|}{ Multivariate analysis } \\
\hline & & Log-rank $x^{2}$ & $P$ & B & SE & HR & $95 \% \mathrm{Cl}$ & $P$ \\
\hline Gender & & 15.660 & $<0.001^{*}$ & 0.194 & 0.150 & 1.190 & $0.905-1.629$ & 0.196 \\
\hline Female & 84.1 & & & & & & & \\
\hline Male & 74.9 & & & & & & & \\
\hline Age (years) & & 38.828 & $<0.001^{*}$ & 0.315 & 0.148 & 1.367 & $1.025-1.833$ & 0.034 \\
\hline$\leq 50$ & 86.1 & & & & & & & \\
\hline$>50$ & 71.4 & & & & & & & \\
\hline Tumor functionality & & 2.821 & 0.093 & & & & & ND \\
\hline Nonfunctional & 77.1 & & & & & & & \\
\hline Functional & 85.2 & & & & & & & \\
\hline Tumor location & & 82.903 & $<0.001^{*}$ & & & & & 0.460 \\
\hline Stomach & 64.6 & & & NA & NA & 1 & Reference & NA \\
\hline Pancreas & 75.3 & & & 0.297 & 0.288 & 1.070 & $0.766-2.366$ & 0.301 \\
\hline Small intestine & 75.8 & & & 0.264 & 0.203 & 1.699 & $0.875-1.939$ & 0.192 \\
\hline Appendix & 86.0 & & & 0.456 & 0.279 & 1.665 & $0.913-2.726$ & 0.103 \\
\hline Colon & 48.5 & & & 0.216 & 0.213 & 1.227 & $0.817-1.883$ & 0.311 \\
\hline Rectum & 90.2 & & & 0.697 & 0.494 & 2.988 & $0.762-5.292$ & 0.159 \\
\hline Tumor size (cm) & & 170.024 & $<0.001^{*}$ & & & & & 0.174 \\
\hline$<2.0$ & 92.6 & & & NA & NA & 1 & Reference & NA \\
\hline $2.0-4.0$ & 71.0 & & & 0.082 & 0.247 & 1.061 & $0.669-1.760$ & 0.740 \\
\hline$>4.0$ & 55.7 & & & 0.364 & 0.263 & 1.411 & $0.859-2.408$ & 0.167 \\
\hline Grade (WHO 2010 classification) & & 325.954 & $<0.001^{*}$ & & & & & $<0.001$ \\
\hline G1 & 93.1 & & & NA & NA & 1 & Reference & NA \\
\hline G2 & 82.7 & & & 0.379 & 0.229 & 1.489 & $0.933-2.287$ & 0.097 \\
\hline G3 & 43.1 & & & 1.627 & 0.221 & 5.172 & $3.301-7.837$ & $<0.001$ \\
\hline Tumor type & & 336.619 & $<0.001^{*}$ & & & & & ND \\
\hline NET & 90.6 & & & & & & & \\
\hline NEC & 42.1 & & & & & & & \\
\hline MANEC & 37.9 & & & & & & & \\
\hline T category (ENETS criteria) & & 204.865 & $<0.001^{*}$ & & & & & 0.401 \\
\hline $\mathrm{T} 1$ & 94.7 & & & NA & NA & 1 & Reference & NA \\
\hline $\mathrm{T} 2$ & 81.6 & & & 0.190 & 0.307 & 1.189 & $0.663-2.206$ & 0.536 \\
\hline T3 & 60.1 & & & 0.454 & 0.308 & 1.594 & $0.862-2.878$ & 0.140 \\
\hline $\mathrm{T} 4$ & 51.3 & & & 0.456 & 0.344 & 1.563 & $0.804-3.097$ & 0.185 \\
\hline N category (ENETS criteria) & & 189.400 & $<0.001^{*}$ & 0.430 & 0.156 & 1.552 & $1.132-2.086$ & 0.006 \\
\hline No & 88.2 & & & & & & & \\
\hline N1 & 53.8 & & & & & & & \\
\hline M category (ENETS criteria) & & 255.266 & $<0.001^{*}$ & 0.784 & 0.164 & 2.107 & $1.590-3.018$ & $<0.001$ \\
\hline MO & 87.2 & & & & & & & \\
\hline M1 & 46.8 & & & & & & & \\
\hline TNM stage (ENETS criteria) & & 332.157 & $<0.001^{*}$ & & & & & ND \\
\hline । & 96.0 & & & & & & & \\
\hline$\|$ & 87.3 & & & & & & & \\
\hline
\end{tabular}

one hand, ENETS guidelines recommend that debulking surgery may be considered for patients with nonfunctional and advanced-stage NETs if the disease does not progress over 6 months and for patients who are suffering from symptoms related to tumor burden [18]. On the other hand, in the context of patients with advanced metastatic NECs, debulking or cytoreductive surgery of primary tumors and surgical resection of metastases are not recommended [18]. Other ablative strategies for liver metastasis are also discouraged [18]. This means that surgery may 
Table 3 continued

\begin{tabular}{|c|c|c|c|c|c|c|c|c|}
\hline \multirow[t]{2}{*}{ Variable } & \multirow[t]{2}{*}{ 3-year OS rate (\%) } & \multicolumn{2}{|c|}{ Univariate analysis } & \multicolumn{5}{|c|}{ Multivariate analysis } \\
\hline & & Log-rank $x^{2}$ & $P$ & B & SE & HR & $95 \% \mathrm{Cl}$ & $P$ \\
\hline III & 64.0 & & & & & & & \\
\hline IV & 46.8 & & & & & & & \\
\hline Surgery & & 212.543 & $<0.001^{*}$ & 0.521 & 0.175 & 1.730 & $1.195-2.370$ & 0.003 \\
\hline Yes & 84.4 & & & & & & & \\
\hline No & 39.3 & & & & & & & \\
\hline
\end{tabular}

OS overall survival, $B$ beta value, $S E$ standard error, $H R$ hazard ratio, $C l$ confidence interval, ND not detected, NA not applicable

* Variables with $P$ value less than 0.05 entered into multivariate analyses, except for tumor type and TNM stage

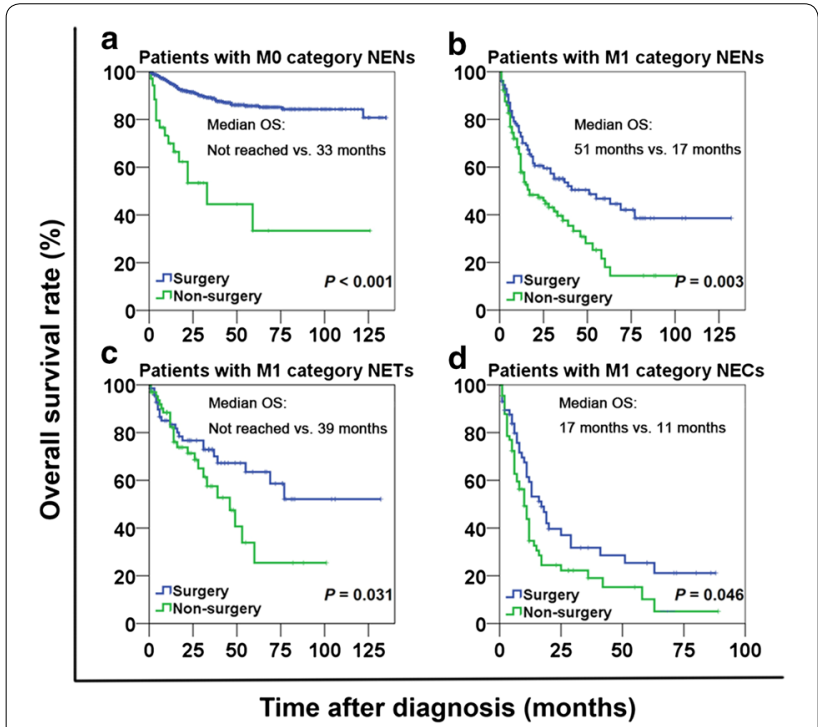

Fig. 2 Patients with neuroendocrine neoplasms (NENs) who underwent surgery had longer OS than those who did not. a OS curves stratified by treatment in patients with MO category NENs. b OS curves of patients with M1 category NENs. c OS curves of patients with M1 category NETs. $\mathbf{d}$ OS curves of patients with M1 category NECs. In the above subgroups, OS is always significantly longer in patients who underwent surgery than in those who did not (all $P<0.05)$

be important for strictly selected patients with advancedstage NECs, such as patients with low tumor burden and limited distant metastases. The survival benefits showed in our study might also be explained by the fact that most advanced-stage cases were comprehensively reviewed by a multidisciplinary team which may select patients with lower metastatic tumor burden and relatively longer stable disease period or patients with better physical condition. Nevertheless, since this study included a limited number of patients with stage IV disease, prospective randomized controlled trials are needed to validate the benefit from surgery and to determine the time point for surgery in patients with advanced-stage disease in clinical practice.
Our study had several limitations. First, data from the five large hospitals cannot be representative of the entire country of China. However, each of these hospitals has different sets of patients. For example, patients with a tumor mass would probably go to the specialized cancer center; those with mild symptoms or who have normal physical examinations would probably go to one of the general hospitals. Thus, because of the inclusion of patients with different characteristics, results of this multicenter study may be representative. Second, since GEPNENs are rare heterogeneous tumors, case numbers in specific subgroups such as patients who undergo different surgical and systematic treatments, are limited, and data could not be statistically analyzed. Third, since the diseases of most patients were diagnosed in the past 5 years, the follow-up duration in our study may be not long enough because these patients usually have a better prognosis compared with patients with other malignancies.

\section{Conclusions}

Our results showed that surgery increased the survival of patients with distant metastasis, even of patients with NECs. However, establishing the optimal criteria to select advanced patients to undergo surgery in clinical practices requires high-level evidence from randomized controlled trials.

\section{Authors' contributions}

$J C, Z Z, Y C, Y L$, and MC contributed to conception and design of the study; CF, WW, YZ, XF, JS, and YZ collected data and consulted literature; CF and WW analyzed data and drafted the manuscript. All authors read and approved the final manuscript.

\section{Author details}

${ }^{1}$ Department of Gastric Surgery, Sun Yat-sen University Cancer Center, State Key Laboratory of Oncology in South China, Collaborative Innovation Center for Cancer Medicine, 651 Dongfeng Road East, Guangzhou 510060, Guangdong, P. R. China. ${ }^{2}$ Department of Gastroenterology, The First Affiliated Hospital of Sun Yat-sen University, Guangzhou 510080, Guangdong, P. R. China. ${ }^{3}$ Department of General Surgery, Guangdong General Hospital, Guangdong Academy of Medical Science, Guangzhou 510080, Guangdong, P. R. China.

${ }^{4}$ Department of Biliopancreatic Surgery, The Sun Yat-sen Memorial Hospital of Sun Yat-sen University, Guangdong Provincial Key Laboratory of Malignant Tumor Epigenetics and Gene Regulation, Guangzhou 510120, Guangdong, P. R. China. ${ }^{5}$ Department of Gastrointestinal Surgery, The Sun Yat-sen Memorial 
Hospital of Sun Yat-sen University, Guangzhou 510120, Guangdong, P. R. China. ${ }^{6}$ Department of Gastroenterology, Nanfang Hospital of Southern Medical University, Guangdong Provincial Key Laboratory of Gastroenterology, Guangzhou 510515, Guangdong, P. R. China.

\section{Acknowledgements}

This work was supported by the Natural Science Foundation of Guangdong Province (No. 2015A030313089), the Medical Science and Technology Research Foundation of Guangdong Province (No. B2014160), and the Major Program of Collaborative Innovation of Guangzhou (No. 201508030042).

\section{Competing interests}

The authors declare that they have no competing interests.

Received: 30 December 2016 Accepted: 3 May 2017

Published online: 21 June 2017

\section{References}

1. Modlin IM, Oberg K, Chung DC, Jensen RT, de Herder WW, Thakker RV, et al. Gastroenteropancreatic neuroendocrine tumours. Lancet Oncol. 2008;9(1):61-72

2. Obendorfer S. Karzinoide tumoren des dunndarms. Frankf Z Pathol. 1907;1:425-9.

3. Bosman F, Carneiro F, Hruban R, Theise N. WHO classification of tumours of the digestive system. 4th ed. Lyon: International Agency for Research on Cancer; 2010.

4. Yao JC, Hassan M, Phan A, Dagohoy C, Leary C, Mares JE, et al. One hundred years after "carcinoid": epidemiology of and prognostic factors for neuroendocrine tumors in 35,825 cases in the United States. J Clin Oncol. 2008;26(18):3063-72.

5. Hauso O, Gustafsson BI, Kidd M, Waldum HL, Drozdov I, Chan AK, et al. Neuroendocrine tumor epidemiology: contrasting Norway and North America. Cancer. 2008;113(10):2655-64.

6. Lepage C, Rachet B, Coleman MP. Survival from malignant digestive endocrine tumors in England and Wales: a population-based study. Gastroenterology. 2007;132(3):899-904.

7. Garcia-Carbonero R, Capdevila J, Crespo-Herrero G, Diaz-Perez JA, Martinez Del Prado MP, Alonso Orduna $V$, et al. Incidence, patterns of care and prognostic factors for outcome of gastroenteropancreatic neuroendocrine tumors (GEP-NETs): results from the National Cancer Registry of Spain (RGETNE). Ann Oncol. 2010;21(9):1794-803.

8. Scherubl H, Streller B, Stabenow R, Herbst H, Hopfner M, Schwertner C, et al. Clinically detected gastroenteropancreatic neuroendocrine tumors are on the rise: epidemiological changes in Germany. World J Gastroenterol. 2013;19(47):9012-9.

9. Lim T, Lee J, Kim JJ, Lee JK, Lee KT, Kim YH, et al. Gastroenteropancreatic neuroendocrine tumors: incidence and treatment outcome in a single institution in Korea. Asia Pac J Clin Oncol. 2011;7(3):293-9.

10. Wang $Y H$, Lin $Y$, Xue L, Wang JH, Chen $M H$, Chen J. Relationship between clinical characteristics and survival of gastroenteropancreatic neuroendocrine neoplasms: a single-institution analysis (1995-2012) in South China. BMC Endocr Disord. 2012;12:30.

11. Jiao X, Li Y, Wang H, Liu S, Zhang D, Zhou Y. Clinicopathological features and survival analysis of gastroenteropancreatic neuroendocrine neoplasms: a retrospective study in a single center of China. Chin J Cancer Res. 2015;27(3):258-66.
12. Rindi G, Kloppel G, Alhman H, Caplin M, Couvelard A, de Herder WW, et al. TNM staging of foregut (neuro)endocrine tumors: a consensus proposal including a grading system. Virchows Arch. 2006;449(4):395-401.

13. Rindi G, Kloppel G, Couvelard A, Komminoth P, Korner M, Lopes JM, et al. TNM staging of midgut and hindgut (neuro)endocrine tumors: a consensus proposal including a grading system. Virchows Arch. 2007:451(4):757-62

14. Modlin IM, Lye KD, Kidd M. A 5-decade analysis of 13,715 carcinoid tumors. Cancer. 2003:97(4):934-59.

15. Ito T, Sasano H, Tanaka M, Osamura RY, Sasaki I, Kimura W, et al. Epidemiological study of gastroenteropancreatic neuroendocrine tumors in Japan. J Gastroenterol. 2010;45(2):234-43.

16. Faggiano A, Ferolla P, Grimaldi F, Campana D, Manzoni M, Davi MV, et al. Natural history of gastro-entero-pancreatic and thoracic neuroendocrine tumors. Data from a large prospective and retrospective Italian epidemiological study: the NET management study. J Endocrinol Invest. 2012;35(9):817-23.

17. Tsai HJ, Wu CC, Tsai CR, Lin SF, Chen LT, Chang JS. The epidemiology of neuroendocrine tumors in Taiwan: a nation-wide cancer registry-based study. PLoS ONE. 2013;8:e62487.

18. Ramage JK, Ahmed A, Ardill J, et al. Guidelines for the management of gastroenteropancreatic neuroendocrine (including carcinoid) tumours (NETs). Gut. 2012;61(1):6-32.

19. Turaga KK, Kvols LK. Recent progress in the understanding, diagnosis, and treatment of gastroenteropancreatic neuroendocrine tumors. CA Cancer J Clin. 2011;61(2):113-32.

20. Chen Wanqing, Zheng Rongshou, Zeng Hongmei, Zhang Siwei. The incidence and mortality of major cancers in China, 2012. Chin J Cancer. 2016;35:73

21. Chen Yong-Sheng, Chen Jian-Guo, Zhu Jian, Zhang Yong-Hui, Ding Lu-Lu. Long-term survival trends of gastric cancer patients between 1972 and 2011 in Qidong. Chin J Cancer. 2015;34:57.

22. Niederle MB, HackI M, Kaserer K, Niederle B. Gastroenteropancreatic neuroendocrine tumours: the current incidence and staging based on the WHO and European Neuroendocrine Tumour Society classification: an analysis based on prospectively collected parameters. Endocr Relat Cancer. 2010;17(4):909-18.

23. Korse CM, Taal BG, van Velthuysen ML, Visser O. Incidence and survival of neuroendocrine tumours in the Netherlands according to histological grade: experience of two decades of cancer registry. Eur J Cancer. 2013;49(8):1975-83.

24. Rothenstein J, Cleary SP, Pond GR, Dale D, Gallinger S, Moore MJ, et al. Neuroendocrine tumors of the gastrointestinal tract: a decade of experience at the Princess Margaret Hospital. Am J Clin Oncol. 2008;31(1):64-70.

25. Ruiz Tovar J, Badia JM. Prevention of surgical site infection in abdominal surgery. A critical review of the evidence. Cir Esp. 2014;92(4):223-31.

26. Aahlin EK, Tranø G, Johns N, Horn A, Søreide JA, Fearon KC, et al. Risk factors, complications and survival after upper abdominal surgery: a prospective cohort study. BMC Surg. 2015;15:83.

27. Ro Cynthia, Chai Wanxing, Yu Victoria E, Run Yu. Pancreatic neuroendocrine tumors: biology, diagnosis, and treatment. Chin J Cancer. 2013;32(6):312-24. 